\title{
COVID-19, Retroelements, and Aging
}

\author{
R. N. Mustafin ${ }^{a, *}$ and E. K. Khusnutdinova ${ }^{b}$ \\ ${ }^{a}$ Bashkir State Medical University, Ufa, 450008 Russia \\ ${ }^{b}$ Institute of Biochemistry and Genetics, Subdivision of the Ufa Federal Research Center, \\ Russian Academy of Sciences, Ufa, 450054 Russia \\ *e-mail: rectorat@bashgmu.ru \\ Received May 17, 2020; revised July 3, 2020; accepted July 7, 2020
}

\begin{abstract}
The review presents an analysis of the scientific data on the characteristics of COVID-19 from the perspective of potential interactions between the virus and the host genome retroelements. According to global statistical data, severe COVID-19 with immune-system hyperactivity is observed mainly in elderly people. At the same time, aging is characterized by a decrease in immune responses. This paradox may be resolved by the assumption that DNA regions that can move along the genome with the "copy and paste" mechanism (retroelements) may play a role in COVID-19 development; these elements are characterized by abnormal expression patterns in aging. Their interaction with SARS-CoV-2 may occur at the level of RNA interference or RNA recombination, or the virus can use retroelement proteins to integrate into the host genome. There is supporting evidence of this interaction: data indicating the efficiency of antiretroviral drugs at the early stage of COVID-19, the isolation of SARS-CoV-2 for a long time after recovery, the persistence of coronavirus infections, and changes in the L1 retrotransposon expression patterns in the lung tissues of COVID-19 patients. In additional, retroelements affect the functioning of the immune system and affect the receptors interacting with SARS-CoV-2. Recombination with retroelements and viral insertions into host genomes have been demonstrated in the case of other infections caused by nonretroviral, RNA-containing viruses. The presumable interaction between SARS-CoV-2 and retroelements may explain the differences in the severity and lethality of COVID-19 in different countries as a result of specific insertional patterns in the genomes of individuals belonging to different human populations. The possible insertion of SARS-CoV-2 cDNA into the genome should be kept in mind in the design of anti-COVID-19 vaccines. Peptide preparations are the most promising in this regard.
\end{abstract}

Keywords: vaccine, peptides, aging, COVID-19, SARS-CoV-2

DOI: $10.1134 / \mathrm{S} 2079057021010458$

\section{INTRODUCTION}

As early as 1962-1967, seroepidemiological studies demonstrated that coronaviruses (CoVs) may be the causative agents of up to $50 \%$ of all cases of seasonal, cold-related diseases [46]. Most of these diseases were characterized as light and moderate acute respiratory infections. However, in 2002, SARS-CoV caused an outbreak of a more severe disease named Severe Acute Respiratory Syndrome (SARS). The epidemic resulted in 8000 cases with A case fatality rate of $9.6 \%$ [54]. From 2012 to 2019, the MERS-CoV coronavirus was the causative agent of a far more severe respiratory syndrome, Middle East Respiratory Syndrome (MERS), with a case fatality rate of $34 \%$. There have been 2458 recorded MERS cases worldwide [4]. In mid-December 2019, a new coronavirus disease characterized by rapid spreading was first recorded in Wuhan, China. WHO experts announced the deciphering of the pathogenic genome on January 12, 2020 , as a result of a study of the virus isolated from the nasopharyngeal cavity of a single patient. It was tenta- tively designated 2019-nCoV. On February 11, 2020, a research group in the International Committee on Taxonomy of Viruses named the new virus SARS-CoV and called the disease it caused Coronavirus Disease2019 (COVID-19) [58].

Coronaviruses are single-stranded, positive-sense, RNA viruses with a genome length that varies within the range of $27-32 \mathrm{~kb}$ and a virus-particle size of $100-$ $160 \mathrm{~nm}$. The Coronaviridae family comprises four genera, Alphacoronavirus, Betacoronavirus, Gammacoronavirus, and Deltacoronavirus. SARS-CoV-2, SARS-CoV, and MERS-CoV belong to the Betacoronavirus genus. The infectious process may involve the respiratory system, as well as the digestive tract. Four COVID-19 clinical course types can be described according to disease severity (Fig. 1) [68]. Bats, e.g., Rhinolophus affinis, are the natural reservoir of SARSCoV-2. Analysis of the codon-usage patterns of SARS-CoV and that of 102367 mammalian species did not confirm the hypothesis that snakes are an intermediate host of this virus [70]. Children develop 


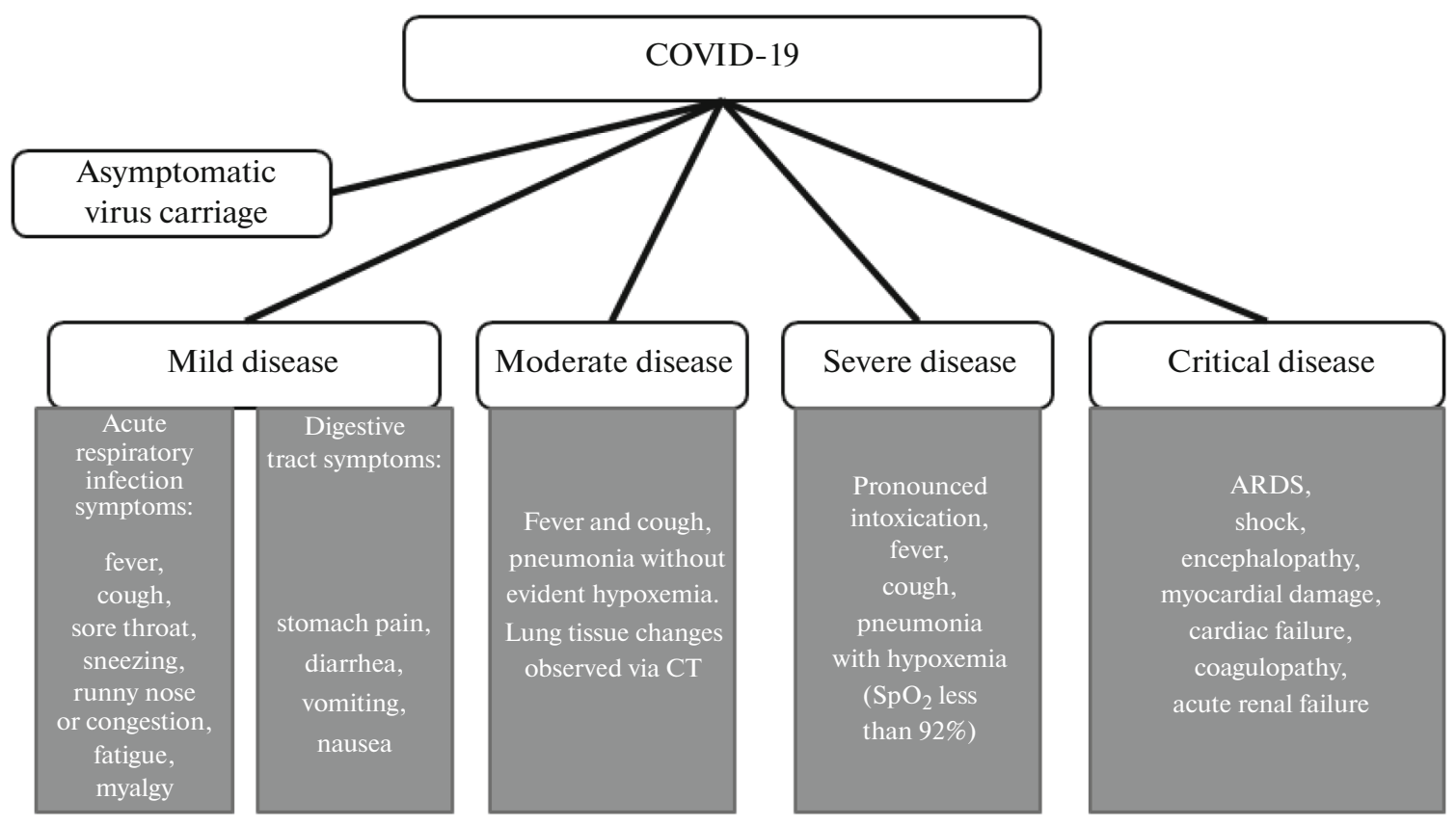

Fig. 1. Classification of COVID-19 clinical types. $\mathrm{SpO}_{2}$ - peripheral oxygen saturation; ARDS-acute respiratory distress syndrome.

COVID-19 less frequently than adults, with $50.9 \%$ of children in China having proved to be virus carriers, $38.8 \%$ demonstrating a mild disease course, and only $5.9 \%$ showing a severe disease course [22]. In adults, the percentage of asymptomatic COVID-19 forms may vary from $1 \%$ in China [8] to $30.8 \%$ and more in other countries [52]. As of May 17, 2020, 4771215 COVID-19 cases have been recorded globally, with the case severity varying significantly from country to country. As a result, the disease lethality also varies considerably based on the official data (coronavirusmonitor.ru). For example, the COVID-19 case fatality rate in Russia is $0.94 \%, 2.78 \%$ in Turkey, $9.96 \%$ in Spain, $15.39 \%$ in France, and $16.38 \%$ in Belgium. What is more, people over the age of 60 years were observed to be more susceptible to SARS-CoV-2 and to develop more serious illness [33] with hyperactive immune responses $[20,26,68]$ despite the decrease in these responses observed with aging $[18,40]$. The goal of the present work is to determine the possible causes of these specific COVID-19, features, which may lay the grounds for the prediction of safe and promising means of disease prevention.

\section{SARS-CoV-2 CHARACTERISTICS}

SARS-CoV-2 is $96.2 \%$ identical to the bat coronavirus $\mathrm{BCoV}$ and $80.26 \%$ identical to SARS-CoV [11]. The life cycle of all CoVs within the host organism includes five stages: attachment, entry, biosynthesis, maturation, and release. Structurally, SARS-CoV-2 virus particles (Fig. 2) contain the following proteins: spike (S), nucleocapsid (N), envelope (E), and membrane (M). The spikes determine the CoV diversity and host tropism. They consist of a transmembrane glycoprotein, which protrudes from the virus surface. The S protein consists of two subunits: $\mathrm{S} 1$, which is responsible for virus binding with the host cell receptor, and S2, which enables virus fusion with cytolemma. The functional receptor for both SARS-CoV and SARS-CoV-2 is the angiotensin-converting enzyme (ACE2) receptor. This determines the CoV pathogenesis, since high levels of ACE2 expression is characteristic of the lungs, heart, large intestine, kidney, and bladder [68]. However, SARS-CoV-2 binds more weakly with ACE2 [67]. The coronavirus S2 subunit contains heptad repeat regions HR1 and HR2, which form a structure that is necessary for membrane fusion. The $\mathrm{E}$ protein plays a pivotal role in virus assembly, budding, and intracellular virus transport. The $\mathrm{M}$ protein is involved in morphogenesis and interaction with other virus components. The S, E, and $\mathrm{M}$ proteins are transported across the rough endoplasmic reticulum membrane into the Golgi apparatus, where they interact with $\mathrm{N}$ proteins and form viral particles [51]. The SARS-CoV-2 genome is $29900 \mathrm{nt}$ long; two thirds of it at the $5^{\prime}$ end is encompassed by the orflab open reading frame. The orf1 polyprotein, which results from the translation of this open reading frame, is cleaved into ten separate nonstructural proteins. The second part of the SARS-CoV-2 genome at the 3'end is represented by the genes encoding the $\mathrm{S}, \mathrm{N}, \mathrm{E}$, and $\mathrm{M}$ structural proteins and the additional ORF3a, ORF6, ORF7a, ORF7b, and ORF8 protein products 


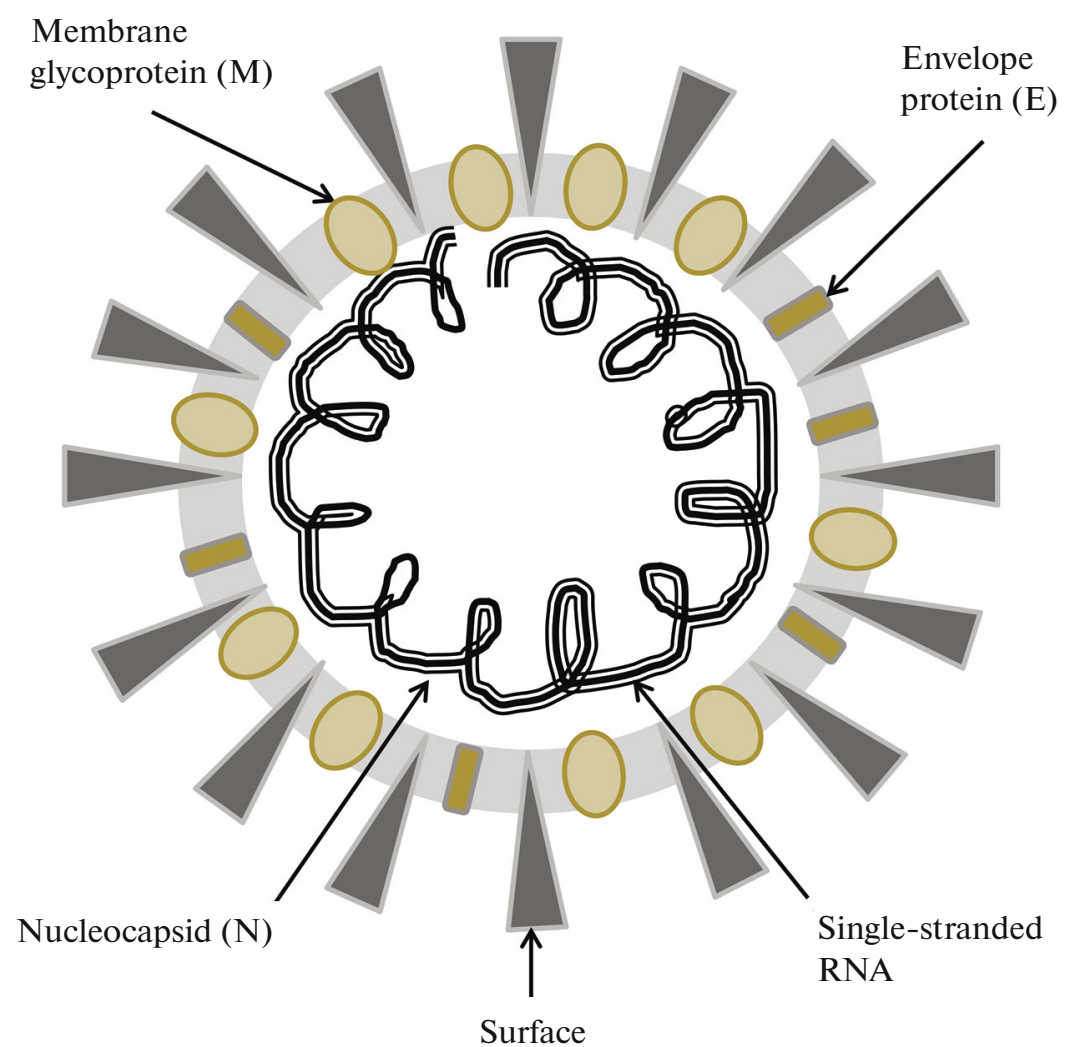

glycoprotein (S)

Fig. 2. SARS-CoV-2 virus particle structure.

(Fig 3) [37]. Unlike SARS-CoV, the SARS-CoV-2 virus also encodes orf8 and orf3b (inhibits interferon beta (IFN $\beta$ ) proteins [67]. It was demonstrated that the SARS-CoV-2 $S$ gene contains four unique insertions that showed a clear similarity to nucleotide sequences in the HIV-1 genome. Three of these insertions were also found in the rat RaTG13 coronavirus. Modeling of the S-protein 3D structure revealed that these insertions are located outside the receptor-binding domains of the spike [70]. In additional, comparison of SARS-CoV-2 nucleotide sequences with the GenBank database revealed many regions that appeared to be identical to some regions in the human genome and the genomes of various animals, as well as HIV-1 [66]. This indicates that there may be recombination between CoVs and cellular RNAs (which is typical of other nonretroviral, RNA-containing viruses (NRRCVs) [3, 24, 25]) and CoVs insertions into host genomes, which was demonstrated for NRRCVs [25, $41,60]$ with the preservation of this insertion in evolution $[5,36]$.

CoVs effectively evade innate immune system responses, which is manifested by a low IL-1 expression in coronavirus infections. CoV-encoded endonucleases (ENs) plays an important role in this process, preventing the activation of double-stranded RNA sensors, such as OligoAdenylate Synthetase (OAS) and pattern-recognition receptors, including Melanoma Differentiation-Associated protein 5 (MDA5) and Protein Kinase RNA-activated (PKR). Infection with EN-deficient coronaviruses leads to immediate IFN-1 activation in the cell and significant attenuation of $\mathrm{CoV}$ replication [39]. At the same time, the OAS-RNase L system is employed to regulate the activity of retroelements (REs), such as L1 [69], while RE transcripts affect PKR and other pattern-recognition receptors, including NOD-like (NLRP3 inflammasome), Toll-like (TLRs), and RIG-1-like receptors (RLRs) [50] that interact with SARS-CoV-2 [20].

\section{CHARACTERISTICS OF THE ANTIVIRAL IMMUNE RESPONSE IN COVID-19}

In the human organism, SARS-CoV-2 enters typeII pneumocytes via the ACE2 receptor [26]. The key components of the innate immune system in the respiratory tract are epitheliocytes, alveolar macrophages (localized in the apical part of the epithelium), and dendritic cells (localized under the epithelium). Virusinfected cells are phagocytosed by macrophages and dendritic cells, which present the antigen to T-lymphocytes. CD4+ T-helpers cause B-lymphocyte activation in order to produce virus-specific antibodies. CD8+ T-killers destroy the infected cells [68]. The 


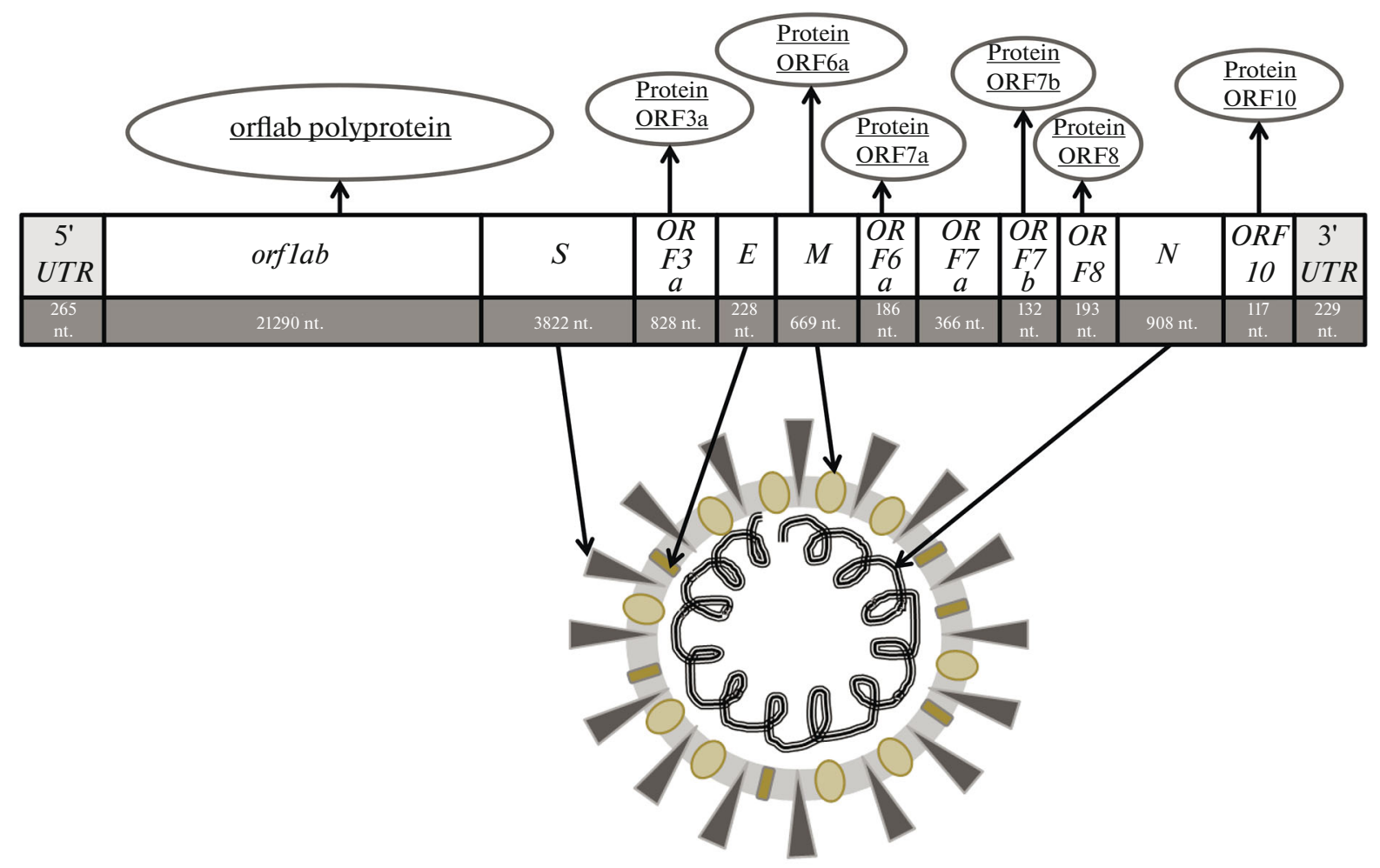

Fig. 3. SARS-CoV-2 genome structure (nt-nucleotide number).

interaction between SARS-CoV-2 and NLRP3, RLR, and TLR stimulate NF- $\mathrm{KB}$ (nuclear factor kappa-B) and interferon regulatory factors 7 and 3 (IRF7 and IRF3). This activates the production of IFN and proinflammatory cytokines and also results in activation of the JAK-STAT signaling cascade, which induces IFN-stimulated genes [20]. An accelerated disease progression in COVID-19 patients is associated with pathological processes in the immune system when inflammatory markers, including interleukins IL-2, IL-7, IL-10, GSCF (granulocyte colonystimulating factor), IP10 (interferon- $\gamma$-inducible protein 10), MCP1 (monocyte chemoattractant protein-1), MIP1 (macrophage inflammatory protein), and TNF- $\alpha$ (tumor necrosis factor $\alpha$ ), are released; their levels are considerably higher than those in patients with mild disease severity [26]. Abnormal hyperproduction of interferons, IL-1, IL-6, and IL-8 is typically observed [20]. An hyperactivated immune response in the case of severe COVID-19 is also characterized by the presence of pathogenic Th1 cells ( $\mathrm{T}$ helpers) and inflammatory monocytes [26].

TLR2 is directly induced by the coronavirus S protein. The E protein has a stimulatory effect on the NLRP3 inflammasome, which leads to increased IL$1 \beta$ production [20]. In addition to EN [39], the virusencoded $\mathrm{N}$ protein is also involved in SARS-CoV-2 evasion of the immune system [67]. In severe COVID19 patients, lymphopenia, in particular, T-cell lymph- openia, is observed with the characteristic activation of not only T-helpers but also T-killers (enhanced expression of CD69, CD38, and CD44). In addition, lung infiltration by high amounts of inflammatory cells, including neutrophils, is observed in the case of severe COVID-19; like a double-edged sword, this induces damage to lungs themselves. This process is also facilitated by the pathological, cytotoxic T-cells derived from T-helpers. The systemic inflammatory process is enhanced by the CD14+CD16+ monocytes, which are activated by GM-CSF and are characterized by increased IL-6 expression. Since SARSCoV-2 binds with ACE2, type-2 and -3 innate lymphoid cells, which also express this receptor, play a certain role in the hyperactive immune response. The thromboses observed in severe COVID-19 cases may also be associated with the presence of ACE2 on endotheliocytes. This massive proinflammatory response, the so-called cytokine storm, may result in the development of acute respiratory distress syndrome and multiple organ dysfunction [68].

\section{RELATIONSHIP OF RETROELEMENTS AND RNA INTERFERENCE WITH CORONAVIRUS INFECTION}

The immune responses that protect against viral infections decrease with age [40]. Clear differences in the T- and B-lymphocyte populations and their functions arise with age; they altogether result in humoral 
and cellular immunity suppression [18]. This does not agree with the predominance of severe COVID-19 forms associated with a hyperactive immune response $[26,68]$ in elderly patients $[8,33]$. One possible explanation for this may be the effects of REs, an imbalance in which is observed in aging [23] and cardiovascular diseases and diabetes mellitus [10]. A severe COVID19 course is also observed in the case of the latter two conditions. As supporting evidence, the study of clinical samples and postmortem lung tissue samples obtained from SARS patients revealed autoantibodies against the EN enzyme of L1 elements in $40.9 \%$ of cases, while an increased expression of L1 EN was detected in lung tissues [28]. The role of enhanced L1 expression in the pathogenesis of autoimmune diseases, such as systemic lupus erythematosus and Sjogren's syndrome, was also demonstrated; the effect is mediated via interferon stimulation [45]. In addition, the antiretroviral drugs Lopinavir and Ritonavir have been shown to be efficient in the treatment of SARS [14] and COVID-19 (combined with Oseltamivir [16] or in the early disease stages [34]).

SARS-CoV-2 can interact with REs at the level of RNA interference (RNAi), since transposons are an important source of noncoding RNAs [1]. This is manifested by changes in the expression of various microRNAs, including those associated with inflammation in aging [65]. In order to counteract RNAi system, SARS-CoV and SARS-CoV-2 N and 7a proteins suppress RNAi. It was demonstrated in experiments with the 293T cell culture that SARS-CoV-2 N protein interacts with the host double-stranded RNAs and exhibits nonspecific, RNA-binding activity that inhibits IFN production. SARS-CoV-2 viruses induce immune responses of varying intensity as a result of their effects on RNAi [50]. This may affect the activity of specific REs associated with immune-system functioning. Hence, organisms' responses may differ depending on the expression pattern of certain REs, especially one takes into account the already established immune imbalance caused by changes in RE activity. For example, L1 in aging cells facilitates inflammatory processes by stimulating IFN-1. At the same time, reverse transcriptase (RT) inhibitors may suppress IFN-1 activation and inflammation in tissues [19]. the role of endogenous retroviruses (ERVs) as IFN-induced enhancers, which are involved in the regulation of key immune functions, including AIM2 inflammasome activation, has been developed. ERVderived regulatory sequences are used by host genomes as a dynamic reservoir of IFN-induced enhancers, which stimulate genetic changes in the mammalian immune system [15].

A study using bronchioalveolar stem cells revealed that coronavirus $\mathrm{N}$ and $\mathrm{S}$ proteins may suppress miR223 and miR-98 expression, thereby controlling the activation of inflammatory chemokines [44]. Experiments using SARS-CoV-2-infected mice demonstrated that viral nucleotide sequences can form com- plementary, palindromic, small RNAs (cpsRNA) $19 \mathrm{nt}$ in length, which cause cell apoptosis [43], as well as small, viral RNAs 18-22 nt in length, which stimulate inflammation in lung tissue [49]. Since there is a strong resemblance between SARS-CoV-2 and SARS$\mathrm{CoV}$ [11], similar mechanisms may be involved in COVID-19 pathogenesis.

It was demonstrated that REs regulate immune system functions. RE transcripts exert effects on TLR, RLR, NLRP3, and protein kinase R (PKR), which recognize viral RNAs during infection [50]. Patternrecognition receptors TLR, RLR, and NLRP3 interact with the RNA products and proteins of human endogenous retroviruses (HERVs), which are similar to virus-encoded proteins. As a result, the signaling cascade associated with the activation of proinflammatory effector genes is activated. Therefore, an RE imbalance can lead to autoimmune reactions and malignant tumors [27]. REs are essential components of immune homeostasis maintenance. Vaccination induces the activation of RE-containing transcripts that silence the IFN promoter in the absence of immune stimuli [29]. The effects of REs on the development of the immune response are mediated not only by the products of their own transcription and translation but also by the specific insertions of these elements into the regions containing genes encoding key immune system factors, thereby regulating their activity. For example, the insertion of HERV-K (HML10) into intron 9 of the $C 4 A$ gene of the class-III main histocompatibility complex has been preserved in evolution [27].

The supporting evidence for the important role played by REs in immune-system responses to viral infections suggests that specific COVID-19 features and the variation in disease lethality indices in different countries may be related to the population-specific insertional characteristics of REs in the human genome, which was demonstrated in several studies [55, 56, 61]. Comparative analysis of the HERV-K distribution in human genomes found 12 insertions that were found to be polymorphic in human populations [61]. The study of polymorphic transposon insertions in 16192 loci in 2504 individuals from 26 populations revealed differences in the insertions that depended on the population's geographical localization [55]. The population-specific distribution of mobile elements in the human genome was been demonstrated when 14384 insertions were examined in 1511 individuals belonging to 15 populations [56]. The same kind of analysis of the genomes of COVID19 patients with different levels of disease severity may provide information on the role specific REs may play in disease severity.

Specific proteins exert effects on the interaction between SARS-CoV-2 and the RNAi system. They may also affect COVID-19 pathogenesis. For example, in the case of SARS-CoV, interaction between 


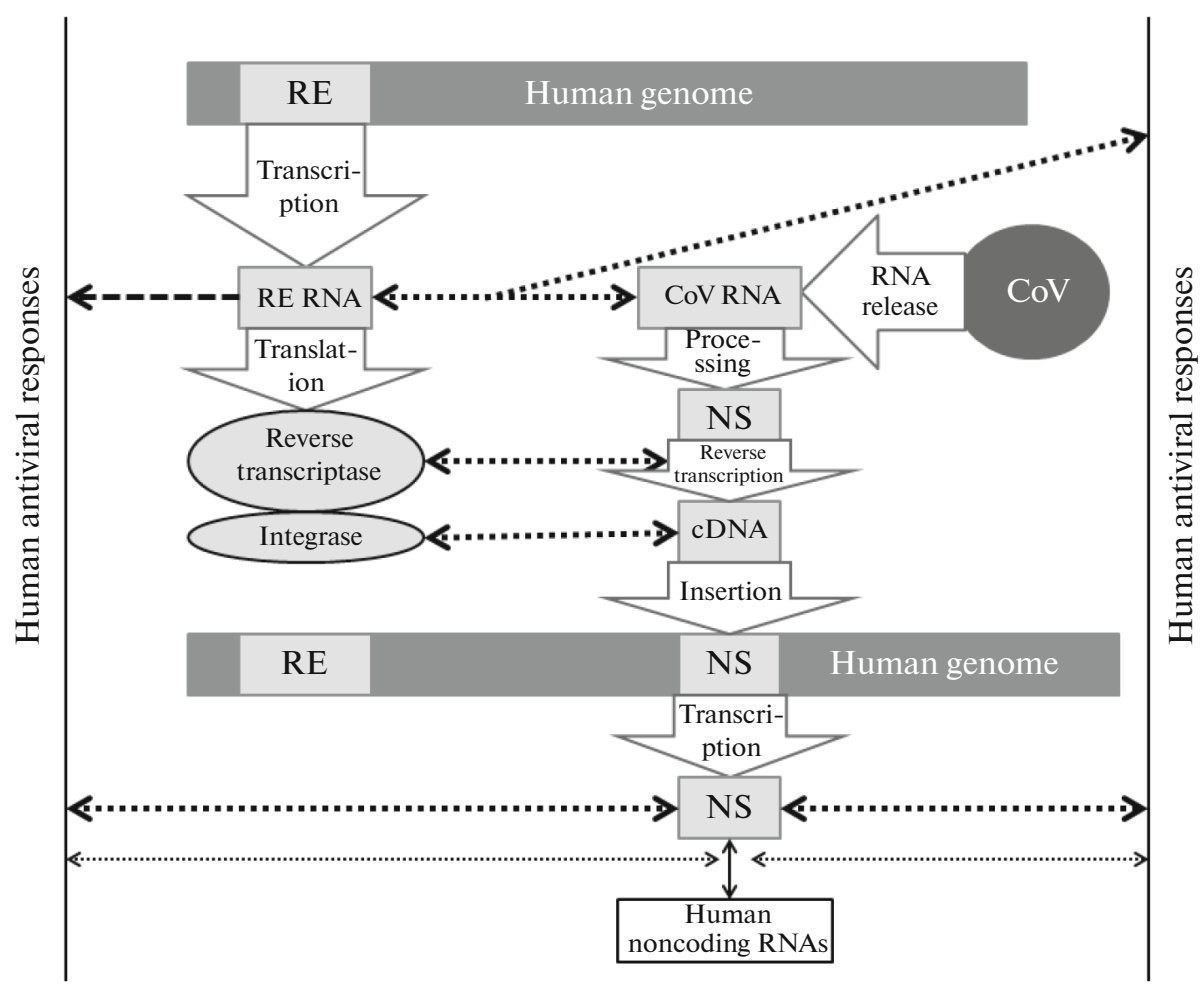

Fig. 4. Schematic outline of the potential involvement of retroelements (REs) in COVID-19 pathogenesis (NS-nucleotide sequences, RE-retroelement).

ezrin and the viral S protein was described, which made it possible to develop a technique to inhibit ezrin expression [48]. The use of target therapy based on small interfering RNAs is suggested due to the inhibitory effects of the SARS-CoV-2 $\mathrm{N}$ and 7 a proteins on the RNAi system [50]. This approach was also developed with the goal of treating MERS [62] and SARS$\mathrm{CoV}$ [9]. For the targeted therapy of SARS-CoV-2, it is predicted that miR-1307-3p and miR-3613-5p miRNAs can prevent viral replication by targeting the 3'-UTR of the virus genome [12]. This approach is highly specific. In particular, studies performed in India identified nine different human miRNAs targeting SARS-CoV-2 genes that lacking targets in the genomes of SARS-CoV and MERS-CoV, with hsalet-7a, hsa-miR101, -125a-5p, -126, -222, -23b, -378, $-380-5$, and -98 among them. What is more, miR-27b proved to be uniquely targeted to mutant SARS-CoV-2 sequences typical of COVID-19 in India. Interestingly, hsa-let-7a, hsa-miR126, hsa-miR378, and hsamiR98 appeared to be also associated with virus hepatitis $\mathrm{C}$; their target in the human organism is IFN $\beta$ gene. MicroRNA hsa-miR101 is associated with herpes simplex virus type- 1 infection, while hsa-miR23b is associated with enterovirus-71 [57]. Figure 4 presents the schematic outline of the possible involvement of REs in COVID-19 pathogenesis.

\section{RELATIONSHIP BETWEEN RETROELEMENTS AND OTHER RNA-CONTAINING VIRUSES}

Studies of other coronaviruses similar to SARSCoV-2 may elucidate the yet unknown COVID-19 pathogenesis pathways leading to severe after-effects of infection. For example, the study of clinical blood samples of SARS patients revealed autoantibodies against the EN enzyme of L1 elements in $40.9 \%$ of cases, while an increased expression of L1 EN was detected in postmortem lung tissue samples [28]. EN is a RE-encoded enzyme that is required for the integration of the element into the genome [23]. It was shown that this protein can be employed by NRRCVs for their insertion [25, 41, 53, 64]. These processes have taken place along the entire course of eukaryotic evolution $[5,30,36,63]$ and may be related to antiviral defense [31, 36, 64]. A similar relationship between SARS-CoV-2 and REs may take place in COVID-19 pathogenesis. The virus may integrate into the human genome with RT and EN, as demonstrated for other positive-strand viruses [5]. This may account for the long-term, post-infection immunity [42] after COVID-19 recovery and the more severe disease forms observed in elderly patients and patients with comorbidities, for whom pathological RE activation is described [10, 23]. Recombination between the virus and cellular RNAs, such as described in the case of NRRCVs [3, 23, 25], is also possible, and it may shed light on the mechanisms of SARS-CoV-2 origination 
from other coronaviruses and explain the presence of sequences shared with the human genome [66].

As early as in 1997, Klenerman et al. demonstrated reverse transcription of NRRCVs with the use of the retroelement-encoded RT upon mouse infection with lymphocytic choriomeningitis virus as an example [41]. In 2009, Geuking et al. showed not only the ability of NRRCVs to integrate into the mouse genome but also the illegitimate recombination between the virus and REs with the same virus as an example [25]. The same process was also described for positivestrand NRRCVs, e.g., the cattle diarrhea virus [3, 24]. The 293T human cell culture was used to demonstrate the reverse transcription of vesicular stomatitis NRRCVa with L1 [60]. Integration may lead to persistent viral infection and the expression of viral peptides, which is taken advantage of by the host for the long-term preservation of immune memory after recovery [31]. This may explain the long-term preservation of genome-integrated NRRCV sequences in evolution due to their adaptive significance for the immune system. The presence of NRRCV insertions in the genomes of various animal species was reported in several independent studies [5, 17, 30, 36, 63]. Genome integration was demonstrated for positivestrand RNA viruses, the replication of which takes place in the cytoplasm as it does with CoVs $[5,36]$.

Viral isolation from patients as late as 14 days after recovery may indicate the importance of interactions between SARS-CoV-2 and RE [42]. Moreover, CoVs infections may result in viral persistence in the host organism [6], which is characteristic of viral integration in the host genome [53]. It was demonstrated for other NRRCVs that viral isolation after recovery was a consequence of viral integration into the genome [31, $53,64]$. This fact should be taken into account when it comes to vaccine development, since the insertion of SARS-CoV-2 sequences into the genome may have yet unpredictable consequences. In this view, peptide vaccines may be considered to be the most safe and the most promising.

\section{PROSPECTS OF DEVELOPING AN ANTI-SARS-CoV-2 VACCINE}

Polypeptide vaccines effectively activate human Tlymphocytes but show poor biological stability. They are characterized by quick inactivation after injection into the organism, which limits their therapeutic activity in vivo. To overcome these limitations, synthetic biology generates highly stable antigenic mimics with artificial subunits. A platform based on D-amino acid combinatorial chemistry was utilized to develop CD8+ T-lymphocyte agonists. It mirrored the immunogenicity profile of a native epitope produced by influenza virus. These artificial peptides were highly stable in human serum and gastric acid and resistant to physical and enzymatic degradation. Subcutaneous and peroral administration of this vaccine to human- ized mice in vivo conferred protection from subsequent infection with a lethal influenza virus [47].

A vaccine against influenza virus $A+H 1 N 1$ was developed with liposome nanoparticles less than $200 \mathrm{~nm}$ in diameter, which encapsulated $10 \mathrm{~B}$ - and T-cell epitope peptides. This vaccine proved to be efficient in experiments on pigs [21]. A chimeric vaccine based on the influenza virus $\mathrm{A} / \mathrm{H} 1 \mathrm{~N} 1$ protein, which flanks an immunogenic peptide from the MERS-CoV spike protein (amino acids 736-761), was constructed [59]. Vaccines based on specific SARS-CoV-2 proteins were developed [13]. There were reports on the design of peptide vaccines against SARS-CoV-2 using immunogenic epitopes of the spike glycoprotein [7], 3CL hydrolase [32], and N, M, and S proteins [35]. In the development of preparations of this kind, the possibility of specific interaction between the peptides and DNA double helix, which may affect the responses of human organism, should not be overlooked $[2,38]$.

It has been also proposed to study peptides (antimicrobial peptides, AMPs) with the prospect of the development of new approaches to the antiviral therapy of coronavirus infection, since many peptide inhibitors have demonstrated nonspecific activity towards viruses. For example, the RVFV-6 peptide obtained from the Rift Valley fever virus (RVFV) glycoprotein proved to be efficient against EBOV and VSV as well. AMPs are the eukaryotic immune system's first line of defense; they show selective toxicity towards bacteria, fungi, and viruses. These short, positively charged peptides selectively act on the negatively charged pathogen's membrane bilayer, which is rich in lipopolysaccharides and lipoteichoic acid. AMPs may affect coronaviruses by inhibiting fusion (the targets are HR1 and HR2 of the S2 protein), entry, replication, or assembly and release [51].

\section{CONCLUSIONS}

Analysis of the available scientific data made it possible to define some points that indicate that REs may play a role in COVID-19 pathogenesis.

Severe illness with immune-system hyperactivity $[20,26,68]$ is mainly experienced by elderly patients [33], despite the decrease in antiviral immunity observed with age $[18,40]$. This may result from the pathological activation of REs with age [10, 23]. The role of retroelements in the development of inflammatory responses via IFN-1 activation was also demonstrated $[15,19]$. These responses may be blocked by reverse transcriptase inhibitors [19]. These preparations may be suggested for COVID-19 therapy. The efficiency of antiretroviral drugs for the treatment of SARS [14], as well as the early stages of COVID-19 $[16,34]$, has been demonstrated in the clinic.

TLR, MDA5, and PKR [20, 39] play an important role in IFN induction in coronavirus infection. The 
REs participating in the cellular antiviral response interact with the same receptors $[15,50]$.

The relationship between SARS-CoV-2 [50] and SARS-CoV [43, 44, 49] and RNA interference has been uncovered; REs play a significant role in the functioning of the latter system [1].

It was demonstrated that other NRRCVs are able to integrate into the host genome with reverse transcriptase and RE integrase. Positive-strand NRRCV insertions into germ cell genomes may be preserved for a long time in evolution $[25,41,60]$, which may indicate their potential role in the antiviral protective response $[31,36,64]$. Similar processes may take place in the case of SARS-CoV-2, since viral RNAs in COVID-19 may be detected as late as 14 days after recovery [42], while persistent infections have been described for other $\mathrm{CoVs}$ [6], which is characteristic of viral integration into the host genome $[31,53]$. This may also account for the presence of the regions identical to certain human DNA regions in SARS-CoV-2 RNA [66].

The evidence of the recombination of NRRCV and RE sequences and cellular RNAs [3, 24, 25] suggest that similar processes are possible for coronaviruses. These processes may have formed the basis for the origin of SARS-CoV-2.

The marked differences in COVID-19 severity and lethality rates in different countries may be caused by differences in the insertional patterns of REs in the genomes of human individuals of different populations $[55,56,61]$. Hence, comparative analysis of the $\mathrm{RE}$ distribution in the genomes of asymptomatic virus carriers and patients with severe COVID-19 may be used to define the mechanisms of resistance to this infection.

Autoantibodies against the EN of L1 elements were detected in about half of SARS patients, and L1 activation was shown in the lungs of patients who died from SARS [28]. In light of the similarity between SARS-CoV and SARS-CoV-2, it may be suggested that similar processes take place in COVID-19 as well.

Noncoding RNAs regulate REs, which are themselves the sources of most of them [1]. This fact should be taken into consideration in the design of target antiCOVID-19 therapy based on the use of specific microRNAs [50, 57]. Moreover, it was demonstrated that vaccination causes the activation of RE-containing transcripts that suppress IFN activation in the absence of immune stimuli [29]. When it comes to the development of an anti-SARS-CoV-2 vaccine, the possible integration of viral nucleotide sequences into the genome with the participation of REs should be taken into account. For this reason, peptide vaccines are considered to be the most promising and safe. It is important to take advantage of the experience of the use of peptide preparations for the introduction of vaccines of this kind into clinical practice [38].

\section{COMPLIANCE WITH ETHICAL STANDARDS}

No experimentation involving animals or human was performed by any of the authors.

Conflict of interest. The authors declare no conflicts of interest.

\section{REFERENCES}

1. Mustafin, R.N. and Khusnutdinova, E.K., The interaction of transposons with epigenetic factors in aging, Usp. Gerontol., 2017, vol. 30, no. 4, pp. 516-528.

2. Khavinson, V.Kh., Solovyov, A.Yu., and Shataeva, L.K., Molecular mechanism of interaction between oligopeptides and double-stranded DNA, Bull. Exp. Biol. Med., 2006, vol. 141, no. 4, pp. 457-461.

3. Austermann-Busch, S. and Becher, P., RNA structural elements determine frequency and sites of nonhomologous recombination in an animal plus-strand RNA virus, J. Virol., 2012, vol. 86, pp. 7393-7402.

4. Azhar, E.I., Hui, D.S.C., Memish, Z.A., et al., The Middle East respiratory syndome (MERS), Infect. Dis. Clin. North Am., 2019, vol. 33, pp. 891-905.

5. Belyi, V.A., Levine, A.J., and Skalka, A.M., Unexpected inheritance: multiple integrations of ancient bornavirus and ebolavirus/marburgvirus sequences in vertebrate, PLoS Pathog., 2010, vol. 6, p. e1001030.

6. Bergmann, C.C., Lane, T.E., and Stohlman, S.A., Coronavirus infection of the central nervous system: host-virus stand-off, Nat. Rev. Microbiol., 2006, vol. 4, pp. $121-132$.

7. Bhattacharya, M., Sharma, A.R., Patra, P., et al., Development of epitope-based peptide vaccine against novel coronavirus 2019 (SARS-COV-2): immunoinformatic approach, J. Med. Virol., 2020, vol. 92, no. 6, pp. 618-631. https://doi.org/10.1002/jmv.25736

8. Bulut, C. and Kato, Y., Epidemiology of COVID-19, Turk. J. Med. Sci., 2020, vol. 50, pp. 563-570.

9. Cao, Y.L., Wang, Y., Guo, R., et al., Identification and characterization of three novel small interference RNAs that effectively down-regulate the isolated nucleocapsid gene expression of SARS coronavirus, Molecules, 2011, vol. 16, no. 2, pp. 1544-1558.

10. Cardelli, M., Giacconi, R., Malavolta, M., and Provinciali, M., Endogenous retroelements in cellular senescence and related pathogenic processes: promising drug targets in age-related diseases, Curr. Drug Targets, 2016, vol. 17, pp. 416-427.

11. Ceraolo, C. and Giorgi, F.M., Genomic variance of the 2019-nCoV coronavirus, J. Med. Virol., 2020, vol. 92, pp. 522-528.

12. Chen, L. and Zhong, L., Genomics functional analysis and drug screening of SARS-CoV-2, Genes Dis., 2020, vol. 14.

https://doi.org/10.1016/j.gendis.2020.04.002

13. Chen, W.H., Strych, U., Hotez, P.J., and Bottazzi, M.E., The SARS-CoV-2 vaccine pipeline: an overview, Curr. Trop. Med. Rep., 2020, vol. 3, pp. 1-4.

14. Chu, C.M., Cheng, V.C., Hung, I.F., et al., Role of lopinavir/ritonavir in the treatment of SARS: initial vi- 
rological and clinical findings, Thorax, 2004, vol. 59, pp. 252-256.

15. Chuong, E.B., Elde, N.C., and Feschotte, C., Regulatory evolution of innate immunity through co-option of endogenous retroviruses, Science, 2016, vol. 351, pp. $1083-1087$.

16. Costanzo, M., De Giglio, M.A.R., and Roviello, G.N., SARS-CoV-2: recent reports on antiviral therapies based on lopinavir/ritonavir, darunavir/umifenovir, hydroxychloroquine, remdesivir, favipiravir and other drugs for the treatment of the new coronavirus, Curr. Med. Chem., 2020, vol. 16. https://doi.org/10.2174/0929867327666200416131117

17. Crochu, S., Cook, S., Attoui, H., et al., Sequences of flavivirus-related RNA viruses persist in DNA form integrated in the genome of Aedes spp. mosquitoes, $J$. Gen. Virol., 2004, vol. 85, pp. 1971-1980.

18. Crooke, S.N., Ovsyannikova, I.G., Poland, G.A., and Kennedy, R.B., Immunosenescence and human vaccine immune responses, Immun. Ageing, 2019, vol. 16, p. 25.

19. De Cecco, M., Ito, T., Petrashen, A.P., et al., L1 drives IFN in senescent cells and promotes age-associated inflammation, Nature, 2019, vol. 566, no. 7742, pp. 7378.

20. De Wilde, A.H., Snijder, E.J., Kikkert, M., and van Hemert, M.J., Host factors in coronavirus replication, Curr. Top. Microbiol. Immunol., 2018, vol. 419, pp. 142.

21. Dhakal, S., Cheng, X., Salcido, J., et al., Liposomal nanoparticle-based conserved peptide influenza vaccine and monosodium urate crystal adjuvant elicit protective immune response in pigs, Int. J. Nanomed., 2018, vol. 13, pp. 6699-6715.

22. Dong, Y., Mo, X., Hu, Y., et al., Epidemiology of COVID-19 among children in China, Pediatrics, 2020, vol. 145 , no. 6 , p. e20200702.

https://doi.org/10.1542/peds.2020-0702

23. Erichsen, L., Beermann, A., Arauzo-Bravo, M.J., et al., Genome-wide hypomethylation of LINE-1 and Alu retroelements in cell-free DNA of blood is an epigenetic biomarker of human aging, Saudi J. Biol. Sci., 2018, vol. 25, pp. 1220-1226.

24. Gallei, A., Pankraz, A., Thiel, H.J., and Becher, P., RNA recombination in vivo in the absence of viral replication, J. Virol., 2004, vol. 78, pp. 6271-6281.

25. Geuking, M.B., Weber, J., Dewannieux, M., et al., Recombination of retrotransposon and exogenous RNA virus results in nonretroviral cDNA integration, Science, 2009, vol. 323, pp. 393-396.

26. Giwa, A.L., Desai, A., and Duca, A., Novel 2019 coronavirus SARS-CoV-2 (COVID-19): an updated overview for emergency clinicians, Emerg. Med. Pract., 2020, vol. 22, pp. 1-28.

27. Grandi, N. and Tramontano, E., Human endogenous retroviruses are ancient acquired elements still shaping innate immune responses, Front. Immunol., 2018, vol. 9, p. 2039.

28. He, W.P., Shu, C.L., Li, B.A., et al., Human LINE1 endonuclease domain as a putative target of SARS-associated autoantibodies involved in the pathogenesis of severe acute respiratory syndrome, Chin. Med. J., 2008, vol. 121, pp. 608-614.

29. Honda, T., Takemoto, K., and Ueda, K., Identification of a retroelement-containing human transcript induced in the nucleus by vaccination, Int. J. Mol. Sci., 2019, vol. 20, p. E2875.

30. Horie, M., Honda, T., Suzuki, Y., et al., Endogenous non-retroviral RNA virus elements in mammalian genomes, Nature, 2010, vol. 463, pp. 84-87.

31. Hurwitz, J.L., Jones, B.G., Charpentier, E., and Woodland, D.L., Hypothesis: RNA and DNA viral sequence integration into the mammalian host genome supports long-term B cell and T cell adaptive immunity, Viral. Immunol., 2017, vol. 30, pp. 628-632.

32. Jakhar, R., Kaushik, S., and Gakhar, S.K., 3CL hydrolase-based multiepitope peptide vaccine against SARSCoV-2 using immunoinformatics, J. Med. Virol., 2020, vol. 92 , no. 10 , pp. $2114-2123$.

https://doi.org/10.1002/jmv.25993

33. Ji, H.L., Zhao, R., Matalon, S., and Matthay, M.A., Elevated plasmin(ogen) as a common risk factor for COVID-19 susceptibility, Physiol. Rev., 2020, vol. 100, no. 3, pp. 1065-1075.

34. Jiang, H., Deng, H., and Wang, Y., The possibility of using lopinavir/ritonavir (LPV/r) as treatment for novel coronavirus COVID-19 pneumonia: a quick systematic review based on earlier coronavirus clinical studies, Zhonghua Jizhen Yixue Zazhi, 2020, vol. 29, pp. 182186.

35. Kalita, P., Padhi, A.K., Zhang, K.Y.J., and Tripathi, T., Design of a peptide-based subunit vaccine against novel coronavirus SARS-CoV-2, Microb. Pathog., 2020, vol. 145, p. 104236.

36. Katzourakis, A. and Gifford, R.J., Endogenous viral elements in animal genomes, PLoS Genet., 2010, vol. 6, p. e1001191.

37. Khailany, R.A., Safdar, M., and Ozaslan, M., Genomic characterization of a novel SARS-CoV-2, Gene Rep., 2020, vol. 100682. https://doi.org/10.1016/j.genrep.2020.100682

38. Khavinson, V.K., Lin'kova, N.S., and Tarnovskaya, S.I., Short peptides regulate gene expression, Bull. Exp. Biol. Med., 2016, vol. 162, pp. 288-292.

39. Kindler, E., Gil-Cruz, C., Spanier, J., et al., Early endonuclease-mediated evasion of RNA sensing ensures efficient coronavirus replication, PLoS Pathog., 2017, vol. 13, p. e1006195.

40. Kim, C., Jadhav, R.R., and Gustafson, C.E., Defects in antiviral T cell responses inflicted by aging-associated miR-181a deficiency, Cell. Rep., 2019, vol. 29, pp. 2202-2216.

41. Klenerman, P., Hengartner, H., and Zinkernagel, R.M., A nonretroviral RNA virus persists in DNA form, $\mathrm{Na}$ ture, 1997, vol. 390, pp. 298-301.

42. Li, Y., Hu, Y., Yu, Y., et al., Positive result of Sars-CoV2 in faeces and sputum from discharged patient with COVID-19 in Yiwu, China, J. Med. Virol., 2020, vol. 92, no. 10, pp. 1938-1947. https://doi.org/10.1002/jmv.25905

43. Liu, C., Chen, Z., Hu, Y., et al., Complemented palindromic small RNAs first discovered from SARS coronavirus, Genes (Basel), 2018, vol. 9, no. 9, p. E442. 
44. Mallick, B., Ghosh, Z., and Chakrabarti, J., MicroRNome analysis unravels the molecular basis of SARS infection in bronchoalveolar stem cells, PLoS One, 2009, vol. 4, p. e7837.

45. Mavragani, C.P., Sagalovskiy, I., Guo, Q., et al., Expression of long interspersed nuclear element 1 retroelements and induction of type I interferon in patients with systemic autoimmune disease, Arthritis Rheumatol., 2016, vol. 68, pp. 2686-2696.

46. McIntosh, K., Kapikian, A.Z., Turner, H.C., et al., Seroepidemiologic studies of coronavirus infection in adults and children, Am. J. Epidemiol., 1970, vol. 92, no. 6 , pp. $585-592$.

47. Miles, J.J., Tan, M.P., Dolton, G., et al., Peptide mimic for influenza vaccination using nonnatural combinatorial chemistry, J. Clin. Invest., 2018, vol. 128, no. 4, pp. 1569-1580.

48. Millet, J.K. and Nal, B., Investigation of the functional roles of host cell proteins involved in coronavirus infection using highly specific and scalable RNA interference (RNAi) approach, Methods Mol. Biol., 2015, vol. 1282, pp. 231-240.

49. Morales, L., Oliveros, J.C., Fernandez-Delgado, R., et al., SARS-CoV-encoded small RNAs contribute to infection-associated lung pathology, Cell Host Microbe, 2017, vol. 21, pp. 344-355.

50. Mu, X., Ahmad, S., and Hur, S., Endogenous retroelements and the host innate immune sensors, $A d v$. Immunol., 2016, vol. 132, pp. 47-69.

51. Mustafa, S., Balkhy, H., and Gabere, M.N., Current treatment options and the role of peptides as potential therapeutic components for Middle East Respiratory Syndrome (MERS): a review, J. Infect. Publ. Health, 2018, vol. 11, no. 1, pp. 9-17.

52. Nishiura, H., Kobayashi, T., Miyama, T., et al., Estimation of the asymptomatic ratio of novel coronavirus infections (COVID-19), Int. J. Infect. Dis., 2020, vol. 94, pp. 154-155.

53. Olson, K.E. and Bonizzoni, M., Nonretroviral integrated RNA viruses in arthropod vectors: an occasional event or something more, Curr. Opin. Insect. Sci., 2017, vol. 22 , pp. $45-53$.

54. Peiris, J.S., Lai, S.T., Poon, L.L., et al., Coronavirus as a possible cause of severe acute respiratory syndrome, Lancet, 2003, vol. 361, pp. 1319-1325.

55. Rishishwar, L., Tellez Villa, C.E., and Jordan, I.K., Transposable element polymorphisms recapitulate human evolution, Mobile DNA, 2015, vol. 6, p. 21.

56. Rishishwar, L., Wang, L., Wang, J., et al., Evidence for positive selection on recent human transposable element insertions, Gene, 2018, vol. 675, pp. 69-79.

57. Sardar, R., Satish, D., Birla, S., et al., Comparative analyses of SARS-CoV-2 genomes from different geographical locations reveal unique features potentially consequential to host-virus interaction, pathogenesis and clues for novel therapies, Heliyon, 2020, vol. 6, no. 9 , p. e04658.

https://doi.org/10.1016/j.heliyon.2020.e04658

58. She, J., Liu, L., and Liu, W., COVID-19 epidemic: disease characteristics in children, J. Med. Virol., 2020, vol. 92 , no. 7 , pp. $747-754$.

https://doi.org/10.1002/jmv.25807

59. Shehata, M.M., Kandei, A., Mostafa, A., et al., A recombinant influenza A/H1N1 carrying a short immunogenic peptide of MERS-CoV as bivalent vaccine in BALB/c mice, Pathogens, 2019, vol. 8, no. 4, p. E281.

60. Shimizu, A., Nakatani, Y., Nakamura, T., et al., Characterization of cytoplasmic DNA complementary to non-retroviral RNA viruses in human cells, Sci. Rep., 2014, vol. 4, p. 5074.

61. Shin, W., Lee, J., Son, S.Y., et al., Human-specific HERV-K insertion causes genomic variations in the human genome, PLoS One, 2013, vol. 8, p. e60605.

62. Sohrab, S.S., El-Kafrawy, S.A., Mirza, Z., et al., Design and delivery of therapeutic siRNAS: application to MERS-coronavirus, Curr. Pharm. Des., 2018, vol. 24, pp. $62-77$.

63. Taylor, D.J., Leach, R.W., and Bruenn, J., Filoviruses are ancient and integrated into mammalian genomes, BMC Evol. Biol., 2010, vol. 10, p. 193.

64. ter Horst, A.M., Nigg, J.C., Dekker, F.M., and Falk, B.W., Endogenous viral elements are widespread in arthropod genomes and commonly give rise to PIWI-interacting RNAs, J. Virol., 2019, vol. 93, p. e02124-18.

65. Williams, J., Smith, F., Kumar, S., et al., Are microRNAs true sensors of ageing and cellular senescence, Ageing Res. Rev., 2017, vol. 35, pp. 350-363.

66. Xiao, C., Li, X., Liu, S., et al., HIV-1 did not contribute to the 2019-nCoV genome, Emerg. Microbes Infect., 2020, vol. 9, pp. 378-381.

67. Yi, Y., Lagniton, P.N.P., Ye, S., et al., COVID-19: what has been learned about the novel coronavirus disease, Int. J. Biol. Sci., 2020, vol. 16, pp. 1753-1766.

68. Yuki, K., Fujiogi, M., and Koutsogiannaki, S., COVID-19 pathophysiology: a review, Clin. Immunol., 2020, vol. 108427.

https://doi.org/10.1016/j.clim.2020.108427

69. Zhang, A., Dong, B., Doucet, A.J., et al., RNase L restricts the mobility of engineered retrotransposons in cultured human cells, Nucleic Acids Res., 2014, vol. 42, pp. 3803-3820.

70. Zhang, C., Zeng, W., Huang, X., et al., Protein structure and sequence reanalysis of 2019-nCoV genome refutes snakes as its intermediate host. or the unique similarity between its spike protein. insertions and HIV-1, J. Proteome Res., 2020, vol. 19, pp. 1351-1360.

Translated by E. Martynova 